\title{
Opportunities to optimise colistin stewardship in hospitalised patients in South Africa: Results of a multisite utilisation audit
}

\author{
A P Messina, ${ }^{1,2}$ BPharm; A J Brink, ${ }^{3,4}$ MB BCh, MMed (Clin Micro); G A Richards, ${ }^{5}$ MB BCh, PhD, FCP (SA), FRCP; S van Vuuren, ${ }^{1}$ PhD \\ ${ }^{1}$ Department of Pharmacy and Pharmacology, Faculty of Health Sciences, University of the Witwatersrand, Johannesburg, South Africa \\ ${ }^{2}$ Department of Pharmacy, Netcare Hospitals, Johannesburg, South Africa \\ ${ }^{3}$ Department of Clinical Microbiology, Ampath National Laboratory Services, Milpark Hospital, Johannesburg, South Africa \\ ${ }^{4}$ Division of Infectious Diseases and HIV Medicine, Department of Medicine, Faculty of Health Sciences, University of Cape Town, South Africa \\ ${ }^{5}$ Division of Critical Care, Faculty of Health Sciences, University of the Witwatersrand, Johannesburg, and Charlotte Maxeke Johannesburg \\ Academic Hospital, Johannesburg, South Africa
}

Corresponding author: S van Vuuren (sandy.vanvuuren@wits.ac.za)

\begin{abstract}
Background. Colistin is an old antibiotic that has been reintroduced as salvage therapy in hospitalised patients because it is frequently the only agent active against Gram-negative bacteria. Various guidelines for colistin administration have led to confusion in establishing the appropriate dose, which has potential for adverse consequences including treatment failure or toxicity. The emergence and spread of colistin resistance has been documented in South Africa (SA), but no local information exists on how and why colistin is used in hospitals, and similarly, compliance with current dosing guidelines is unknown.

Objectives. To evaluate the current utilisation of colistin in SA hospitals, in order to identify stewardship opportunities that could enhance the appropriate use of this antibiotic.

Methods. Electronic patient records of adult patients on intravenous (IV) colistin therapy for $>72$ hours in four private hospitals were retrospectively audited over a 10-month period (1 September 2015 - 30 June 2016). The following data were recorded: patient demographics, culture and susceptibility profiles, diagnosis, and indication for use. Compliance with six colistin process measures was audited: obtaining a culture prior to initiation, administration of a loading dose, administration of the correct loading dose, adjustments to maintenance dose according to renal function, whether colistin was administered in combination with another antibiotic, and whether de-escalation following culture and sensitivity results occurred. Outcome measures included effects on renal function, overall hospital mortality, intensive care unit length of stay (LoS), and hospital LoS.

Results. Records of 199 patients on IV colistin were reviewed. There was $99.0 \%$ compliance with obtaining a culture prior to antibiotic therapy, $93.5 \%$ compliance with prescription of a loading dose, and $98.5 \%$ compliance regarding prescription of colistin in combination with another agent. However, overall composite compliance with the six colistin stewardship process measures was $82.0 \%$. Non-compliance related to inappropriate loading and maintenance doses, lack of adjustment according to renal function and lack of de-escalation following culture sensitivity was evident. Significantly shorter durations of treatment were noted in patients who received higher loading doses $(p=0.040)$ and in those who received maintenance doses of $4.5 \mathrm{MU}$ twice daily v. $3 \mathrm{MU}$ three times daily $(p=0.0027)$. In addition, compared with patients who survived, more patients who died received the $3 \mathrm{MU}$ three times daily maintenance dose ( $p=0.0037$; phi coefficient 0.26 ). Conclusions. The study identified multiple stewardship opportunities to optimise colistin therapy in hospitalised patients. Urgent implementation of a stewardship bundle to improve colistin utilisation is warranted.
\end{abstract}

S Afr Med J 2018;108(1):28-32. DOI:10.7196/SAMJ.2018.v108i1.12561

The emergence of life-threatening multidrug-resistant (MDR) bacteria has been widely documented as a global threat to society, because effective antibiotics to treat bacterial infections are rapidly diminishing. ${ }^{[1]}$ A global review of antibiotic consumption revealed that antibiotic utilisation increased by $36 \%$ over a 10 -year period (2000 - 2010), with the most notable escalation reflected in the carbapenem and polymyxin classes. ${ }^{[2]}$ This trend correlates with the mounting rates of MDR and extensively drug-resistant (XDR) Gramnegative pathogens, which include the carbapenemase-producing Enterobacteriaceae (CPE), which often require the use of older, more toxic drugs such as colistin as last-resort salvage therapy for critically ill patients in hospital settings. ${ }^{[3]}$

Colistin, also known as polymyxin $\mathrm{E}$, is a nephrotoxic and neurotoxic, concentration-dependent bactericidal antibiotic. It became accessible for use in the late 1950s, but its use diminished over time as newer, less toxic agents such as the aminoglycosides became available. ${ }^{[4-5]}$ Unfortunately, the current dosing guidelines for colistin, with regard to both administration and dose, are outdated, inaccurate and confusing, as the package insert information has not been revised since its initial launch. ${ }^{[6]}$ This compromises the management of patients with serious Gram-negative infections and potentially increases resistance. ${ }^{[7]}$ Recommendations also differ between the European and American literature, depending on whether the metric system has been adopted or not. Either international units or milligrams of the sodium salt colistimethate (the inactive prodrug) may be used, and to confuse matters further, colistin base activity (CBA) in milligrams may also be used to define the dose. ${ }^{[4,6]}$ Many believe that the appropriate use of colistin is not known, as 
knowledge of its pharmacokinetics (PK) and pharmacodynamics (PD) are incomplete ${ }^{[8]}$ However, a recent large multicentre PK study involving 215 patients described a novel algorithmic approach for intravenous (IV) colistin dosing, establishing for the first time dosing recommendations based on accurate PK data. ${ }^{[9]}$

Colistin in South Africa (SA) is only authorised in exceptional circumstances following application to and approval from the Medicines Control Council, as stipulated in section 21 of the Medicines and Related Substances Control Act. This process is often associated with delays in drug procurement. ${ }^{[10]}$ Over- or incorrect use of antibiotics can lead to the development of resistance ${ }^{[11]}$ and, while the emergence and spread of colistin resistance, including heteroresistance among CPE (OXA-48 producing Klebsiella pneumoniae) and plasmid-mediated resistance, has recently been documented in $\mathrm{SA},{ }^{[12-14]}$ the degree of compliance with current dosing guidelines is unknown and no local information on why and how colistin is prescribed is available. Colistin hetero-resistance is defined as 'the emergence of resistance to colistin by a subpopulation from an otherwise susceptible (MIC [minimum inhibitory concentration] of $\leq 2 \mathrm{mg} / \mathrm{liter}$ ) population' that may be related to exposure to suboptimal polymyxin concentrations. ${ }^{[14]}$

\section{Objectives}

The main purpose of this study was to evaluate the current utilisation of colistin in four private sector SA hospitals. We also hoped to identify opportunities to improve the appropriate use of colistin in the future, in an attempt not only to improve outcomes, but to negate or minimise the risk of development of resistance in vivo.

\section{Methods}

This multicentre retrospective record review of patients receiving IV colistin treatment was conducted in four private sector SA hospitals, comprising two each in Johannesburg and Pretoria. The necessary approvals for this study were obtained from the individual participating hospitals, and ethical clearance (ref. no. M150404) was granted by the University of the Witwatersrand Human Research Ethics Committee. Hospital names were kept anonymous for ethical reasons.

Data were collected and captured in the electronic Bluebird system (http://www.intelms.com/) for a 10-month period (1 September 2015 - 30 June 2016). This system integrates laboratory data from the main private laboratories with dispensing data and the patient's admission master file. This allowed for the identification of patients in each hospital to whom colistin had been dispensed, and for the monitoring of laboratory culture results, drug prescription data, hospital ward movements and overall patient outcomes. The study included adult patients aged $>18$ years who were prescribed IV colistin for at least 72 hours. Paediatric and neonatal patients and those who received colistin treatment via an alternative route of administration were excluded. Following review of patient records from the Bluebird system, findings were entered onto a spreadsheet using Microsoft Excel version 14 (Microsoft, USA) for statistical analysis and qualitative interpretation.

The following patient information was collected: age, gender, weight $(\mathrm{kg})$, hospital ward location, admission diagnosis, serum creatinine level and estimated glomerular filtration rate (eGFR). The indications for colistin therapy were recorded as follows: empirical therapy (if no evidence of an MDR or XDR Gram-negative organism was found prior to or during the course of treatment), directed therapy (infection with an MDR or XDR organism of known sensitivity) or salvage therapy (failure of an alternative treatment where colistin was used as escalation therapy).

\section{Process and outcome measures}

Audit of colistin process measures included the following: obtaining a culture prior to colistin initiation, prescription of a loading dose, prescription of correct loading and maintenance doses, and adjustments of maintenance doses according to renal function as stipulated by two SA guidelines, ${ }^{[10,15]}$ administration of colistin in combination with another antibiotic, duration of therapy (calculated as number of treatment days), and de-escalation following culture and sensitivity results. First or repeat courses were also documented, and the type of culture specimens, the organisms cultured and the sensitivity profiles were matched to the prescription data. Outcome measures included were effect on renal function, overall hospital mortality, intensive care unit (ICU) length of stay (LoS) and hospital LoS.

\section{Statistical analysis}

The relationship between the duration of therapy and other study variables was assessed by the $t$-test, or one-way analysis of variance (ANOVA) for more than two categories. Where the data did not meet the assumptions of these tests, a non-parametric alternative, the Wilcoxon rank-sum test (or the Kruskal-Wallis test for more than two categories), was used. The $\chi^{2}$ test was used to assess the relationships between patient outcomes and other study variables. Fisher's exact test was used for $2 \times 2$ tables or where the requirements for the $\chi^{2}$ test could not be met. Data analysis was carried out using SAS version 9.4 for Windows (SAS Institute, USA). The 5\% significance level was used.

\section{Results}

A total of 212 patients received IV colistin during the study period. Of these $13 \mathrm{did}$ so for $<72$ hours and were therefore excluded. Table 1 describes the demographics and characteristics of the patients included in the study. The mean age of the patients was 50.9 years (standard deviation (SD) 16.9), range 19 - 93). The most common admission diagnosis was haematological malignancy $(37.2 \%, n=74)$ followed by trauma $(12.1 \%, n=24)$ and then bloodstream infection $(7.5 \%, n=15)$. Laboratory culture identification was undertaken from 197 patients. Blood cultures were the most frequent $(54.3 \%, n=107)$,

Table 1. Demographics and characteristics of the patients studied $(N=199)$

\begin{tabular}{ll}
\hline & Patients, $\boldsymbol{n}(\%)$ \\
\hline Number of patients per hospital & $56(28.1)$ \\
Hospital 1 & $22(11.1)$ \\
Hospital 2 & $17(8.5)$ \\
Hospital 3 & $104(52.3)$ \\
Hospital 4 & $40(20.1)$ \\
Distribution of patients according to hospital location \\
$\quad$ General wards & $159(79.9)$ \\
ICUs & $113(56.8)$ \\
Gender & $86(43.2)$ \\
Male & \\
Female & $163(81.9)$ \\
Course of colistin therapy & $36(18.1)$ \\
First course & \\
Repeat course & $63(31.1)$ \\
Indication for colistin therapy & $121(60.8)$ \\
Empirical therapy & $15(7.5)$ \\
Directed therapy & \\
Salvage therapy &
\end{tabular}


followed by urine $(14.7 \%, n=29)$, sputum $(8.6 \%, n=17)$ and tracheal aspirates $(8.1 \%, n=16)$. The most prevalent organisms necessitating the use of colistin were K. pneumoniae (39.2\%, $n=78)$, Pseudomonas aeruginosa $(20.1 \%, n=40)$ and Acinetobacter baumannii $(9.0 \%, n=18)$. Of these, $77.9 \%(n=106)$ were resistant to the carbapenems. The most commonly co-administered antibiotics were meropenem $(60.7 \%$, $n=119)$ and tigecycline $(28.6 \%, n=56)$. In $63.8 \%(n=125), 32.1 \%$ $(n=63)$ and $4.1 \%(n=8)$ of patients, one, two and three Gram-negative antimicrobial agents, respectively, were prescribed in addition to colistin. The median duration of colistin therapy was 9 days (interquartile range (IQR) 6 - 16, range 3 - 63). Most patients (57.8\%, $n=115)$ received a course of therapy of $\leq 10$ days, $13.6 \%(n=27)$ a course of $11-14$ days and $28.6 \%(n=57)$ a course of $\geq 15$ days.

\section{Process measures}

As demonstrated in Table 2, there was considerable variability in both the prescribed loading and maintenance doses, as well as in frequency of administration. Non-compliance with SA dosing adjustment recommendations according to renal function is depicted in Table 3 , and the audit of compliance with all the colistin process measures is summarised in Table 4.

\section{Outcome measures}

Durations of treatment were found to be significantly shorter in patients who received higher loading doses $(p=0.040)$ (Fig. 1). Those who received maintenance doses of 4.5 MU IV 12-hourly v. $3 \mathrm{MU}$ IV 8-hourly also had shorter durations of therapy, 8 days v. 12 days, respectively ( $p=0.0027)$. The duration of therapy for patients with $P$. aeruginosa infection (median 12 days, IQR 8 - 23) was significantly longer than for those without (median 9 days, IQR 6 - 15) $(p=0.044)$. No significant differences were found in the median duration of therapy for patients in the ICU v. the general wards $(p=0.41)$ or between those with positive blood culture samples v. other specimen types $(p=0.39)$. In addition, no difference was noted regarding treatment duration and overall patient outcome $(p=0.20)$.

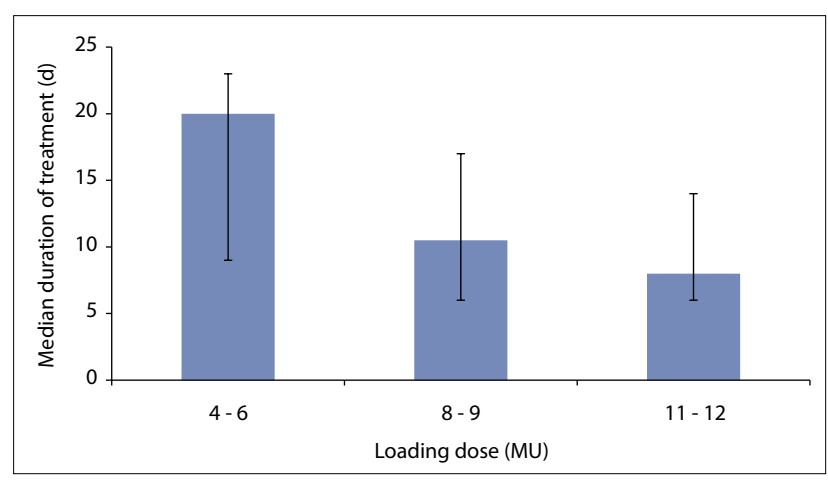

Fig. 1. Associations between colistin loading dose and median duration of treatment. The error bars denote the interquartile range.

\begin{tabular}{|c|c|c|c|c|c|}
\hline LD & $\begin{array}{l}\text { Patients (N=186), } \\
n(\%)\end{array}$ & MD & $\begin{array}{l}\text { Patients }(N=199) \text {, } \\
n(\%)\end{array}$ & $\begin{array}{l}\text { Frequency of } \\
\text { administration }\end{array}$ & $\begin{array}{l}\text { Patients }(N=100), \\
n(\%)\end{array}$ \\
\hline $4 \mathrm{MU}$ & $3(1.6)$ & $1 \mathrm{MU}$ & $3(1.5)$ & 6-hourly & $1(0.5)$ \\
\hline $6 \mathrm{MU}$ & $10(5.4)$ & $1.5 \mathrm{MU}$ & $11(5.5)$ & 8-hourly & $76(38.2)$ \\
\hline $8 \mathrm{MU}$ & $5(2.7)$ & $2 \mathrm{MU}$ & $17(8.5)$ & 12-hourly & $122(61.3)$ \\
\hline $9 \mathrm{MU}$ & $45(24.2)$ & $2.5 \mathrm{MU}$ & $2(1.0)$ & & \\
\hline $11 \mathrm{MU}$ & $1(0.5)$ & $3 \mathrm{MU}$ & $65(32.7)$ & & \\
\hline $12 \mathrm{MU}$ & $122(65.6)$ & $4.5 \mathrm{MU}$ & $101(50.8)$ & & \\
\hline
\end{tabular}

\section{Table 3. Compliance with recommended adjustments of colistin dosage according to renal function}

\begin{tabular}{|c|c|}
\hline \multicolumn{2}{|c|}{ South African Society of Clinical Pharmacy guideline ${ }^{[15]}$} \\
\hline Normal renal function & $\begin{array}{l}\text { Loading dose } 12 \mathrm{MU} \text {, then } 3 \mathrm{MU} \text { 8-hourly } \\
\text { or } 4.5 \mathrm{MU} 12 \text {-hourly }\end{array}$ \\
\hline $\mathrm{CrCl} 40-60 \mathrm{~mL} / \mathrm{min}$ & 2 MU 12-hourly \\
\hline $\mathrm{CrCl} 10-40 \mathrm{~mL} / \mathrm{min}$ & 2 MU 24-hourly \\
\hline $\mathrm{CrCl}<10 \mathrm{~mL} / \mathrm{min}$ & 1.5 MU 36-hourly \\
\hline \multicolumn{2}{|c|}{ Compliance with recommended dosing guidelines for study patients $(N=199), n(\%)$} \\
\hline Unknown* & $58(29.2)$ \\
\hline Compliant & $68(34.2)$ \\
\hline Non-compliant & $73(36.7)$ \\
\hline \multicolumn{2}{|l|}{ Visser Kift et al. guideline ${ }^{[10]}$} \\
\hline Critically ill or severe sepsis & Loading dose 9 - $12 \mathrm{MU}$ \\
\hline $\mathrm{eGFR}>60 \mathrm{~mL} / \mathrm{min}$ & 4.5 MU 12-hourly \\
\hline eGFR $30-60 \mathrm{~mL} / \mathrm{min}$ & 3 MU 12-hourly \\
\hline eGFR $10-30 \mathrm{~mL} / \mathrm{min}$ & 2 MU 12-hourly \\
\hline $\mathrm{eGFR}<10 \mathrm{~mL} / \mathrm{min}$ & 1 MU 12-hourly \\
\hline \multicolumn{2}{|c|}{ Compliance with recommended dosing guidelines for study patients $(N=199), n(\%)$} \\
\hline Unknown ${ }^{*}$ & $3(1.5)$ \\
\hline Compliant & $80(40.2)$ \\
\hline Non-compliant & $116(58.3)$ \\
\hline
\end{tabular}


Table 4. Compliance with colistin stewardship process measures

\begin{tabular}{ll}
\hline & $\begin{array}{l}\text { Compliance rate }(N=199), \\
\text { Process measures }\end{array}(\boldsymbol{n})$ \\
\hline Obtaining an appropriate culture prior to commencement of colistin therapy & $99.0(197)$ \\
Prescription of a loading dose & $93.5(186)$ \\
Prescription of an appropriate loading dose & $90.3(168)$ \\
Prescription of appropriate maintenance dosing, including adjustment according to renal insufficiency & $48.2(68 / 141)^{\star}$ \\
& $40.8(80 / 196)^{\dagger}$ \\
& $98.5(196)$ \\
Prescription of colistin in combination with another Gram-negative antibiotic & $69.9(58 / 83)$ \\
De-escalation of colistin therapy & \\
${ }^{*}$ According to the South African Society of Clinical Pharmacy guideline. ${ }^{[15]}$ &
\end{tabular}

The effects on renal function in patients who received IV colistin therapy were insignificant. There was no change in median creatinine level after receiving colistin $(73 \mu \mathrm{mol} / \mathrm{L}$, IQR 53 - 110, range 21 - 601 before; $73 \mu \mathrm{mol} / \mathrm{L}$, IQR 51 - 128, range 20 - 645 after). Similarly, the mean eGFR was $79 \mathrm{~mL} / \mathrm{min}$ (SD 37, range 8 - 150) before treatment and $79 \mathrm{~mL} / \mathrm{min}$ (SD 38, range 7 - 150) after treatment. Prior to the commencement of colistin, $5.6 \%$ of patients $(n=11)$ were considered to have kidney failure, $7.1 \%(n=14)$ to have severe kidney injury, and $66.8 \%(n=131)$ to have normal kidney function according to the Kidney Disease Improving Global Outcomes (KDIGO) classification.

The median ICU LoS was 31 days (IQR 15 - 52, range 0 - 152) and the median hospital LoS was 46 days (IQR 25 - 83, range 3 227). The majority of the patients $(70.4 \%, n=140)$ were discharged, indicating a $29.6 \%$ in-hospital mortality rate $(n=59)$. This study found no significant association between patient outcomes and a particular organism, the presence or absence of a bloodstream infection, duration of therapy or loading dose. However, a significant association was found between outcome and the maintenance dose prescribed ( $p=0.0037$; phi coefficient 0.26 ). Patients who died had received lower prescribed maintenance doses per interval than those who survived (Fig. 2).

\section{Discussion}

To our knowledge this is the first study that has evaluated the current utilisation of colistin across multiple SA hospitals and involving a large sample of patients. As a result, numerous opportunities for improved stewardship were identified. Recent recommendations in response to the emergence and spread of plasmid-mediated colistin resistance include preserving colistin use for definitive treatment based on susceptibility testing, use of $\mathrm{PK} / \mathrm{PD}$ indicators to ensure appropriate dosing, and use of empirical therapy in selected cases only. ${ }^{[16]}$

Contrary to these recommendations, our study suggests that both loading and maintenance dosing of colistin is variable and inconsistent, with adherence to available local dosing guidelines at best $48.2 \%$. This reveals the extent of uncertainty associated with colistin utilisation in SA hospitals and the very urgent need for education so that our last-resort Gram-negative antibiotic can be preserved for as long as possible. The administration of a colistin loading dose is widely considered to be best practice, as it facilitates the rapid achievement of optimal bactericidal concentrations. ${ }^{[17,18]}$ Although compliance with the recommendation for a loading dose was high (93.5\%), the actual loading doses ranged from $4 \mathrm{MU}$ to 12 MU. This demonstrates a lack of understanding regarding the need for appropriate loading doses, which should be in the region of $9-12$ MU regardless of renal function. ${ }^{[9,19]}$ The findings of our study further emphasise the importance of ensuring optimal dosing as, although Acute Physiology and Chronic Health Evaluation II (APACHE II) scores were not available so that patients could be appropriately

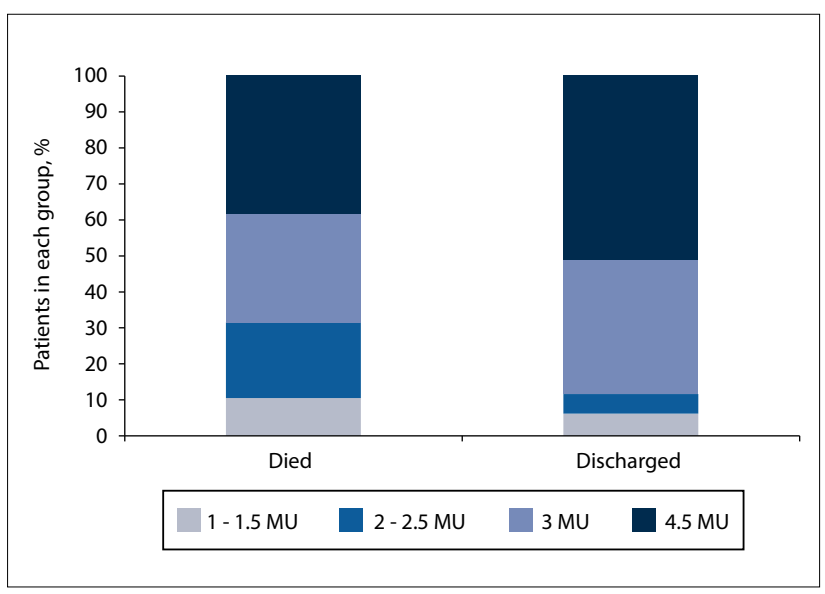

Fig. 2. Associations between colistin maintenance doses and patient outcome.

matched, higher loading doses appeared to be associated with shorter duration of treatment. Interestingly, there also appeared to be an association with the manner in which the maintenance doses were administered. Although most patients received $9 \mathrm{MU} /$ day, those who received doses of 4.5 $\mathrm{MU}$ twice daily also had a shorter duration of therapy and more favourable overall outcomes, which may be due to the concentration-dependent nature of the drug. This is in keeping with the concept that administration of antibiotics according to PK principles and rapid achievement of therapeutic concentrations would result in improved clinical cure. . $17,20-21]^{-1}$

Combination therapy was prescribed to all but three patients in our study. This practice, including duplicate and sometimes triplicate therapy, is recommended by local guidelines for the treatment of CPE, suggesting that combinations may improve efficacy and minimise the risk of selection of resistant organisms. Studies that have supported combination therapy for CPE have relatively low sample sizes, and concerns remain regarding the increased environmental burden of multiple antibiotic exposure, which may actually increase colonisation rates with resistant organisms and increase the risk of Clostridium difficile infection. ${ }^{[2]}$ One recent study could not demonstrate whether it was combination therapy alone rather than higher dosing that contributed to better patient outcomes, albeit in a small patient study population $(N=28) \cdot{ }^{[17]}$ Randomised clinical trials are underway to help establish whether patient outcomes are actually improved with combinations compared with appropriately dosed and administered colistin monotherapy. ${ }^{[1,22]}$ Until these results are available, combination therapy is currently the recommended best practice.

Of concern is the large proportion of patients in our study (31.1\%) who received empirical colistin therapy. This could be due to the expectation of increased MDR and XDR infections in the large population of severely immunocompromised haematology patients 
included in the study, for whom colistin may well be appropriate. For $69.9 \%(n=58)$ of the eligible patients, therapy was de-escalated to a narrower-spectrum antibiotic following the availability of sensitivity results. Although this is a somewhat low figure, it is in line with other studies indicating that de-escalation is not always possible for many reasons, including the limited number of effective antibiotics available to treat MDR infections, the limited understanding of how to de-escalate, and the fact that the practice has still not been widely accepted in critically ill patients. ${ }^{[23]}$

Colistin-related nephrotoxicity remains an important concern and has been found to be influenced by elevated plasma drug concentrations $(>2.5 \mathrm{mg} / \mathrm{L})$ and longer duration of therapy. ${ }^{[24]}$ Similar insignificant effects as in our analysis of renal function have been demonstrated. ${ }^{[17]}$ Another study found that up to $43 \%$ of patients were at risk of or had acute kidney injury or renal failure according to the Risk, Injury, Failure, Loss and End-stage kidney disease (RIFLE) criteria after IV colistin therapy; however, this toxicity was reversed following discontinuation of treatment. ${ }^{[25]}$ Recent results from the multicentre PK colistin study demonstrated that there was huge interpatient variability in the clearance of colistin (even at similar creatinine clearances), which is probably due to differences between individuals in conversion rates of the inactive prodrug to its active form. ${ }^{[9]}$ This adds to the complexity of providing optimal dosing, given the very narrow therapeutic window of the drug.

\section{Study limitations}

Limitations of this study include its retrospective nature and the need to collect data from electronic prescription records, which may not always record parameters such as renal replacement therapy. Furthermore, the high number of patients who did not have their weight recorded made it difficult to calculate creatinine clearances using the Cockcroft-Gault equation. Compliance with the South African Society of Clinical Pharmacy dosing guidelines based on creatinine clearance may therefore be skewed. Although not an objective of the study, illness severity scores such as the APACHE II score were not recorded and patient risk in relation to mortality or outcome could therefore not be corrected. The median duration of colistin therapy in this study was 9 days. In these scenarios, treatment courses are typically determined at the discretion of the prescribing clinician, and this commonly depends on the clinical response to therapy and/or evidence of microbiological cure. As such, duration of therapy could not be used as a process indicator owing to the limited guidance available on what an appropriate duration should be. In addition, the dosing duration may have been a function of prescriber education rather than an indication that higher loading doses were associated with a more rapid clinical response. Finally, data on side-effects of colistin other than nephrotoxicity were not actively investigated.

\section{Conclusions}

This study showed that multiple stewardship opportunities for improvement exist, including administration of appropriate colistin loading and maintenance doses, optimising maintenance doses according to renal function, prioritising culture-driven prescribing where possible, and monitoring of combination therapy, de-escalation practices and duration of treatment to preserve colistin efficacy for the foreseeable future. The introduction of colistin therapeutic drug monitoring would be extremely useful to individualise dosing, given the variability of PK and PD parameters in critically ill patients. As an immediate solution, however, the design of a colistin antimicrobial stewardship bundle to increase composite compliance could have a significant impact and is strongly recommended.
Acknowledgements. We acknowledge the invaluable contributions of the pharmacists at the participating hospitals for their day-to-day management of patients on colistin, and the hospitals and hospital group for participating in the study. We would also like to acknowledge Dr Petra Gaylard from DMSA (Data Management and Statistical Analysis) for her valuable insight and input relating to the statistics and data analysis.

Author contributions. APM, AJB and SvV designed the study. APM conducted the study and collected the data. AJB and SvV are cosupervisors to APM, as the study forms part of a postgraduate degree. GAR provided expert input into the study design. All authors interpreted the data, wrote the article and approved the final version.

Funding. This study was supported by a Faculty Research Committee individual research grant from the Faculty of Health Sciences, University of the Witwatersrand.

Conflicts of interest. None.

1. O'Neill J. Review on AMR. Antimicrobial resistance: Tackling a crisis for the health and wealth of nations. December 2014. https://amr-review.org/sites/default/files/160518_Final\%20paper_with\%20cover.pdf (accessed 17 November 2016).

2. Van Boeckel TP, Gandra S, Ashok A, et al. Global antibiotic consumption 2000 to 2010: An analysis of national pharmaceutical sales data. Lancet Infect Dis 2014;14(8):742-750. https://doi.org/10.1016/s14733099(14)70780-7

3. Goff DA, Kaye KS. Minocycline: An old drug for a new bug: Multi-drug resistant Acinetobacter baumanii. Clin Infect Dis 2014;59(6):365-366. https://doi.org/10.1093/cid/ciu531

4. Biswas S, Brunel J, Dubus J, Reynaud-Gaubert M, Rolain J. Colisitin: An update on the antibiotic of the 21st century. Expert Rev Anti Infect Ther 2012;10(8):917-934. https://doi.org/10.1586/eri.12.78

Pike M, Saltiel E. Colistin- and polymyxin-induced nephrotoxicity: Focus on literature utilizing the RIFLE classification scheme of acute kidney injury. J Pharm Pract 2014;27(6):554-561. https://doi. org/10.1177/0897190014546116

6. Nation RL, Li J, Cars O, et al. Framework for optimisation of the clinical uses of colistin and polymyxin B: The Prato polymyxin consensus. Lancet Infect Dis 2015;15(2):225-234. https://doi.org/10.1016/s14733099(14)70850-3

7. Kassamali Z, Rotschafer JC, Jones RN, Prince RA, Danziger LH. Polymyxins: Wisdom does not always come with age. Clin Infect Dis 2013;57(6):877-883. https://doi.org/10.1093/cid/cit367

8. Ortwine JK, Kaye KS, Li J, Pogue J. Colistin: Understanding and applying recent pharmacokinetic advances. Pharmacother 2014;35(1):11-16. https://doi.org/10.1002/phar.1484

Nation RL, Garoznik SM, Thamlikitkul V, et al. Dosing guidance for intravenous colistin in critically ill patients. Clin Infect Dis 2017;64(5):565-571. https://doi.org/10.1093/cid/ciw839

10. Visser Kift E, Maartens G, Bamford C. Systematic review of the evidence for rational dosing of colistin. S Afr Med J 2014;104(3):183-186. https://doi.org/10.7196/SAMJ.10727

11. Drusano GL, Louie A, MacGowan A, Hope W. Suppression of emergence of resistance in pathogenic bacteria: Keeping our powder dry, Part 1. Antimicrob Agents Chemother 2016;60(3):1183-1193. https:// doi.org/10.1128/AAC.02177-15.

12. Jayol A, Poirel L, Brink AJ, Villegas M, Yilmaz M, Nordmann P. Resistance to colistin associated with a single amino acid change in protein PmrB among Klebsiella pneumoniae isolates of worldwide origin. Antimicrob Agents Chemother 2014;58(8):4762-4766. https://doi.org/10.1128/aac.00084-14

13. Coetzee J, Corcoran C, Prentice E, et al. Emergence of plasmid-mediated colistin resistance (MCR-1) among Escherichia coli isolated from South African patients. S Afr Med J 2016;106(5):449-450. https:/ doi.org/10.7196/samj.2016.v106i5.10710

14. Jayol A, Nordmann P, Brink AJ, Poirel L. Heteroresistance to colistin in Klebsiella pneumoniae associated with alterations in the PhoPQ regulatory system. Antimicrob Agents Chemother 2015;59(5):2780-2784. https://doi.org/10.1128/aac.05055-14

15. Labuschagne Q, Schellack N, Gous A, et al. Colistin: Adult and paediatric guideline for South Africa South Afr J Infect Dis 2016;1(1):1-5. https://doi.org/10.1080/23120053.2016.1144285

16. Al-Tawfiq JA, Laxminarayan R, Mendelson M. How should we respond to the emergence of plasmidmediated colistin resistance in humans and animals? Int J Infect Dis 2017;54:77-84. https://doi org/10.1016/j.ijid.2016.11.415

17. Dalfino L, Puntillo F, Mosca A, et al. High-dose, extended-interval colistin administration in critically ill patients: Is this the right dosing strategy? A preliminary study. Clin Infect Dis 2012;54(12):1720-1726 https://doi.org/10.1093/cid/cis286

18. Garoznik SM, Li J, Thamlikitkul V, et al. Population pharmacokinetics of colistin methanesulfonate and formed colistin in critically ill patients from a multicenter study provide dosing suggestions for variou categories of patients. Antimicrob Agents Chemother 2011;55(7):3284-3294. https://doi.org/10.1128 aac.01733-10

9. Richards GA, Joubert IA, Brink AJ. Optimising the administration of antibiotics in critically ill patients. S Afr Med J 2015;105(5):419. https://doi.org/10.7196/SAMJ.9649

20. Vicari G, Bauer SR, Neuner EA, Lam SW. Association between colistin dose and microbiological outcomes in patients with multidrug-resistant Gram-negative bacteraemia. Clin Infect Dis 2012;56(3):398-404. https://doi.org/10.1093/cid/cis909

21. Falagas M, Rafailidis PI, Ioannidou E, et al. Colistin therapy for microbiologically documented multidrug-resistant Gram-negative bacterial infections: A retrospective cohort study of 258 patients. Int ] multidrug-resistant Gram-negative bacterial infections: A retrospective cohort study of 258
Antimicrob Agents 2010;35(2):194-199. https://doi.org/10.1016/jijantimicag.2009.10.005

22. Paul M, Carmeli Y, Durante-Mangoni E, et al. Combination therapy for carbapenem-resistant Gramnegative bacteria. J Antimicrob Chemother 2014;69(9):2305-2309. https://doi.org/10.1093/jac/dku168 23. Garnacho-Montero J, Escoresca-Ortega A, Fernandes-Delgado E. Antibiotic de-escalation in
the ICU: How is it best done? Curr Opin Infect Dis 2015;28(2):193-198. https://doi.org/10.1097/
qco.0000000000000141

24. Hartzell JD, Neff R, Ake J, et al. Nephrotoxicity associated with intravenous colistin (colistimethate sodium) treatment at a tertiary care medical center. Clin Infect Dis 2009;48(12):1724-1728. https://doi.

25. Pogue JM, Lee J, Marchaim D, et al. Incidence of and risk factors for colistin-associated nephrotoxicity in a large academic health system. Clin Infect Dis 2011;53(9):879-884. https://doi.org/10.1093/cid/cir611 